\title{
Prévalence des hémoparasites chez le tisserin villageois (Ploceus cucullatus) dans la ville de Dschang et ses environs (Ouest-Cameroun)
}

\author{
Ghislain Noé PIEBENG KOUGOUM ${ }^{1 *}$, Simon AWAFOR TAMUNGANG ${ }^{2}$, \\ Catherine FUSI NGWA ${ }^{3}$, Julius Awah NDUKUM ${ }^{4}$ et Josué WABO PONE ${ }^{3}$ \\ ${ }^{I}$ Dépatement des Sciences Environnementales, Institut Supérieur du Sahel, Université de Maroua, BP, 46 \\ Maroua, Cameroun. \\ ${ }^{2}$ Département de Biologie Animale, Faculté des Sciences, Université de Dschang, BP. 146, \\ Dschang, Cameroun. \\ ${ }^{3}$ Département de Biologie Animale, Faculté des Sciences, Université de Dschang, BP. 67 Dschang, Cameroun. \\ ${ }^{4}$ Département de Physiologie et de Production Animale, Faculté d'Agronomie et des Sciences Agricoles, \\ Université de Dschang, BP 222, Dschang, Cameroun. \\ *Auteur correspondant ; E-mail : kougoumghislain@yahoo.fr, Tél : 77381313 ou 97642197
}

\section{RESUME}

Cette étude évalue la prévalence des hémoparasites chez 62 tisserins gendarmes (Ploceus cucullatus) dans la ville de Dschang et ses environs. L'analyse des frottis sanguins de ces oiseaux pour la recherche des hématozoaires a révélé 41 (soit 66,13\%) porteurs d'au moins un genre d'hémoparasites avec par ordre des prévalences Plasmodium et/ou Haemoproteus $(58,06 \%)$; Leucocytozoon $(19,35 \%)$ et Trypanosoma $(4,84 \%)$. Aucune microfilaire n'a été détectée. Pour l'analyse de ces observations, nous avons envisagé l'interaction des différentes variables environnementales (sites de capture et saisons) et les caractéristiques de l'oiseau (âge, sexe et poids). Aucune différence significative n'a été constatée pour les infestations à hémoparasites chez les tisserins en fonction du sexe, de l'âge, du poids, du site de capture et de la saison. Les sites de capture, l'âge et le sexe semblent ne pas influencer la transmission de ces parasites chez le tisserin gendarme. L'ensemble de ces observations permet de mieux comprendre l'interaction tisserin-parasites et soulève bon nombre de questions sur la pathogénicité de ces parasites, leur transmission et les risques qu'ils présentent pour la santé publique.

(C) 2014 International Formulae Group. All rights reserved.

Mots clés : Tisserin gendarme, hématozoaires, transmission, pathogénicité.

\section{INTRODUCTION}

Les oiseaux sont des hôtes d'une très grande diversité de parasites. Parmi ceux-ci figurent les hémoparasites qui infestent tous les vertébrés et peuvent affecter l'évolution et l'écologie de plusieurs espèces (Barroca, 2005). Ces parasites présentent un cycle de développement où alternent les phases sexuées et asexuées réalisées dans des cellules des tissus et du sang de leur hôte (Valkiũnas, 2005). Les infestations à hémoparasites chez les oiseaux sont communes, et on estime que près de $68 \%$ d'espèces d'oiseaux sont susceptibles aux hématozoaires. La transmission de ces parasites est vectorielle et fait intervenir de nombreux arthropodes (Rahimanga et al., 2002). On peut cependant distinguer des familles et des genres de vecteurs en fonction des parasites (Valkiũnas, 2005) : Plasmodium est transmis par des Culicidae (essentiellement des genres Culex, Culiseta, Aedes, Anopheles et Mansonia); 
Haemoproteus est transmis par des Ceratopogonidae et des Hippoboscidae; et Leucocytozoon est transmis par des Simulidae.

Les genres de protozoaires les plus fréquemment rencontrés sont : Haemoproteus, Plasmodium et Leucocytozoon pour les Apicomplexa, Coccidia et Trypanosoma pour les Euglénobiontes. Les microfilaires sanguines pour les nématodes sont également d'observation courante. Les oiseaux constituent aussi des réservoirs d'une part de nombreux agents infectieux (virus, rickettsies, bactéries et champignons microscopiques), et d'autre part, d'arthropodes agents vecteurs des maladies (Murphy, 1998 ; Daszak et al., 2000). A cause de leur grande mobilité, les tisserins villageois constituent un problème de la santé publique. Oiseau à migration locale, le tisserin gendarme est le plus commun et le plus abondant de la famille des Ploceidae. La plupart des individus vivent en colonies bruyantes à proximité des villes et villages (Fry et Keith, 2004). Ils sont en contact permanent avec les humains et d'autres animaux, ce qui peut entraîner un échange de parasites soit par contact direct ou indirectement par l'intermédiaire d'un vecteur.

Bien que la faune aviaire de l'Afrique soit hautement diversifiée, les parasites sanguins de cette avifaune sont peu connus (Sehgal et al., 2005). De nombreuses études pour la recherche des hémoparasites ont déjà été menées dans plusieurs pays d'Afrique, y compris le Cameroun. Dans la majorité des cas, seules les familles d'oiseaux sont prises en considération (Sehgal et al., 2005). Au niveau de l'espèce d'oiseau, dans la plupart des cas, quelques oiseaux seulement ont été échantillonnés. Au cours de la plupart de ces travaux, aucun tisserin gendarme (Ploceus cucullatus) n'avait été capturé pour les prélèvements sanguins. Leurs microorganismes restent également inconnus.

Le but de notre travail était de rechercher les parasites sanguins des tisserins gendarmes à Dschang et d'étudier l'influence des différents facteurs environnementaux (saisons et sites de capture) ou spécifique de l'oiseau (âge, sexe et poids) sur ces parasites. Cette étude participe à l'établissement de l'état de santé de cette avifaune à Dschang, ainsi que sur les risques potentiels de transmission de ces germes à l'homme et aux autres animaux.

\section{MATERIEL ET METHODES \\ Site de l'étude}

Cette étude a été réalisée de novembre 2005 à avril 2006 à Dschang, ville située entre $05^{\circ} 20^{\prime}-07^{\circ} 00^{\prime} \mathrm{N}$ et $10^{\circ} 30-12^{\circ} 00^{\prime} \mathrm{E}$ à $1400 \mathrm{~m}$ d'altitude. Le climat y est tropical de type camerounéen caractérisé par deux saisons : une longue saison pluvieuse de mi-mars à minovembre et une courte saison sèche de minovembre à mi-mars. La végétation est une savane arbustive, avec des palmiers raphia dans la majorité des vallées, dans les marécages ou en bordure des cours d'eau. La pluviométrie annuelle avoisine $2400 \mathrm{~mm}$ pour une humidité relative et une température moyenne annuelle de $72 \%$ et $20,2{ }^{\circ} \mathrm{C}$ respectivement.

\section{Matériel animal}

Soixante cinq (65) tisserins gendarmes ont été capturés sur 6 sites de nidification présents dans la ville de Dschang au début de l'étude (Figure 1). Trois (3) hôtes ont été retrouvés morts le lendemain du jour de leur capture et ne seront pas inclus dans le présent travail. Le nombre d'oiseaux capturés par sites est présenté dans le Tableau 1.

\section{Méthodes de capture des oiseaux}

Les captures d'oiseaux ont été faites à la tombée de la nuit à l'aide d'un piège artisanal confectionné à l'extrémité d'un bambou (Figure 2). Les oiseaux capturés ont été transportés au laboratoire dans des cages individuelles et conservés dans les mêmes conditions au laboratoire.

\section{Sexage des oiseaux}

Compte tenu du fait que chez les tisserins villageois les jeunes mâles ressemblent aux jeunes femelles et aux femelles adultes, le sexe des jeunes oiseaux a été déterminé sur la base de l'anatomie des gonades (testicules chez les mâles et ovaires chez les femelles) après dissection. La taille des ovaires a permis de séparer les jeunes 
femelles de leurs congénères adultes. Les mâles adultes ont quant à eux été identifiés grâce à leur plumage nuptial qu'il garde tout au long de l'année dans les zones tropicales humides (Fry et Keith, 2004). Par la suite, les oiseaux ont été pesés à l'aide d'une balance de marque Metler PE 160, de capacité 210 g ( \pm $0.0001 \mathrm{~g}$ ) et regroupés en deux catégories d'âge : jeunes et adultes.

Prélèvement du sang, confection et coloration des frottis minces

Les prélèvements sanguins ont été effectués à l'aide d'une seringue de 2,5 ml au niveau du cœur sur chaque oiseau anesthésié au chloroforme. Une goutte de ce sang a permis de réaliser un frottis sanguin pour chaque oiseau. Puis, les lames ont été séchées à l'air libre, fixées au May-Grünwald et colorées au Giemsa (OMS, 1982). Chaque lame a été examinée au microscope optique à l'objectif 100x pendant 20 minutes pour la recherche des protozoaires et à l'objectif 40x pour les microfilaires.

L'identification au niveau des genres des hémoparasites s'est faite sur la base des critères tels que la pigmentation du parasite intra-érythrocytaire (Plasmodium), présence des gamétocytes pigmentés et non pigmentés pour les genres Haemoproteus et Leucocytozoon respectivement, présence du flagelle (Trypanosome) et présence ou l'absence de gaine chez les microfilaires.

\section{Analyses statistiques}

La comparaison des prévalences moyennes en fonction des sites de capture, de l'âge, du sexe, du poids et de la saison était faite en utilisant le test exact de Fisher au seuil de probabilité $\mathrm{P}=0,05$.

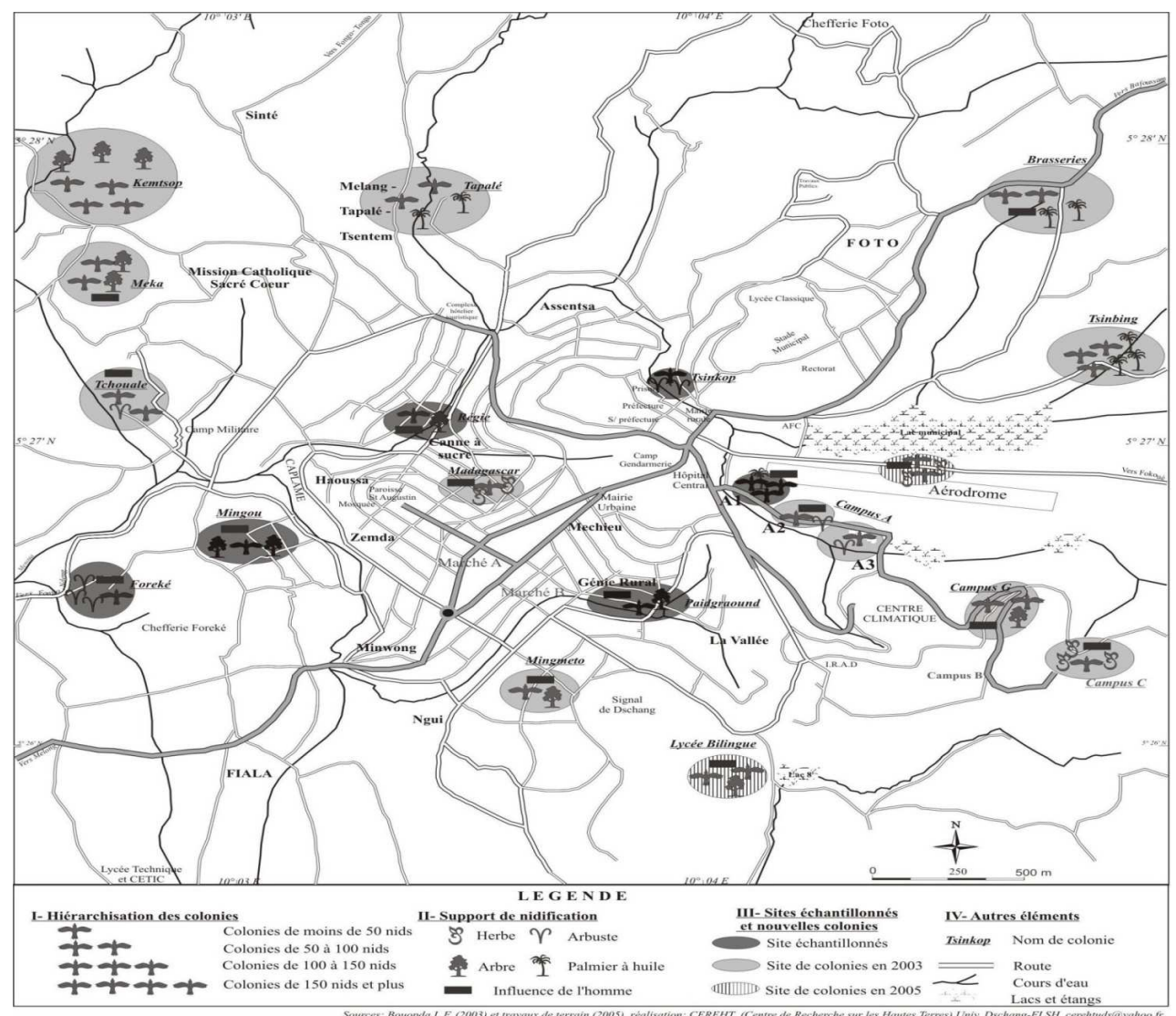

Figure 1 : Répartition des colonies de tisserin et sites d'échantillonnage dans la ville de Dschang et ses environs. 
Tableau 1 : Dates et nombre d'oiseaux capturés par site.

\begin{tabular}{|c|c|c|c|}
\hline Sites de capture & Dates de capture & & Nombre d'oiseaux \\
\hline Foreké & $\begin{array}{l}\text { 18-25 Nov } 2005 \\
07 \text { Mar } 2006\end{array}$ & $\begin{array}{l}7 \\
1\end{array}$ & 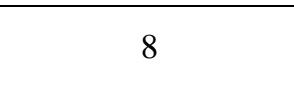 \\
\hline Tsinkop & $\begin{array}{l}\text { 17-26 Nov } 2005 \\
\text { 05-23 Fév } 2006 \\
26 \text { Avr } 2006\end{array}$ & $\begin{array}{l}7 \\
4 \\
1\end{array}$ & 12 \\
\hline Régie & $\begin{array}{l}17 \text { Nov } 2005 \\
\text { 07-23 Fév } 2006 \\
26 \text { Mar } 2006 \\
28 \text { Avr } 2006 \\
\end{array}$ & $\begin{array}{l}1 \\
10 \\
3 \\
2 \\
\end{array}$ & 16 \\
\hline Campus A & $\begin{array}{l}27 \text { Jan } 2006 \\
\text { 02-23 Fév } 2006 \\
08 \text { et } 27 \text { Mar } 2006 \\
15 \text { et } 18 \text { Avril } 2006\end{array}$ & $\begin{array}{l}1 \\
9 \\
5 \\
2\end{array}$ & 17 \\
\hline Mingouh & 09-12 Mar 2006 & 7 & 7 \\
\hline Paidground & $\begin{array}{l}\text { 23 Mar } 2006 \\
18-24 \text { Avr } 2006\end{array}$ & $\begin{array}{l}2 \\
3\end{array}$ & 5 \\
\hline Total & & & 65 \\
\hline
\end{tabular}

\section{RESULTATS}

Soixante-deux (62) oiseaux ont fait l'objet des prélèvements pour la confection des frottis sanguins. Parmi ces frottis, 41 (soit $66,13 \%)$ se sont révélés positifs par au moins un genre d'hémoparasite. Les parasites identifiés se repartissent de la façon suivante : 36 Plasmodium /Haemoproteus $(58,06 \%) ; 12$ Leucocytozoon (19,35\%); 3 Trypanosoma $(4,84 \%)$ et 1 Plasmodium $(1,61 \%)$ sur la base d'un schizonte (Tableau 2). Aucune microfilaire n'a été détectée.

La différenciation des genres Plasmodium et Haemoproteus n'a pas été possible lorsque les frottis mettaient en évidence seulement les gamétocytes. Ainsi, nous avons mis ensemble les deux genres (Plasmodium/Haemoproteus). Les prévalences des hémoparasites selon les sites de capture sont consignées dans le Tableau 2.

Il ressort de ce tableau que les oiseaux ont été plus infestés à Mingouh (85,71\%) suivi de ceux du campus A $(68,75 \%)$. Les oiseaux des sites de la Régie et Foréké ont présenté des prévalences d'infestation égales $(62,50 \%)$. Il en est de même de ceux de Tsinkop et Paidground (60\%). Toutefois, aucune différence significative n'a été observée entre les prévalences moyennes globales des différents sites de capture $(\mathrm{P} \geq 0,05)$. Le genre Trypanosoma a été identifié chez les oiseaux des sites de Régie et de Mingouh avec des prévalences moyennes très proches $(12,50$ et $14,29 \%$ respectivement). Les parasites identifiés dans le présent travail ont présenté des prévalences variées en fonction des sites de capture. Par exemple, le groupe Plasmodium /Haemoproteus et les trypanosomes sont plus prévalents à Mingouh avec des fréquences moyennes respectives de $85,71 \%$ et $14,29 \%$. Les infestations à Leucocytozoon sont plus observées à la régie $(32,25 \%)$. Toutefois, aucune différence significative $(P \geq 0,05)$ n'a été observée entre les prévalences moyennes des infestations à Plasmodium $s p /$ Haemoproteus $s p$ et celles à Leucocytozoon $s p$ dans les différents sites de capture. 
Influence de l'âge et du sexe sur la distribution des hémoparasites

Quels que soient l'âge et le sexe des oiseaux, ceux-ci sont infestés par les trois genres de parasites identifiés. Les jeunes étant plus infestés par les genres Leucocytozoon et Trypanosoma $(23,53 \%$ vs $17,78 \%$ et $11,76 \%$ vs $2,22 \%$ respectivement) alors que, les adultes le sont par le groupe Plasmodium/ Haemoproteus (60\% vs 52,94\%) (Tableau 3). Dans le même ordre d'idées, en considérant le sexe des oiseaux, seuls les femelles hébergent les trypanosomes tandis que les mâles sont plus infestés par les autres genres (Plasmodium /Haemoproteus, Leucocytozoon) (65,38\% et $23,08 \%$ respectivement) comparés aux femelles $(52,78 \%$ et $16,67 \%$ respectivement) (Tableau 3). Il n'y a aucune différence significative $(\mathrm{P} \geq 0,05)$ entre les prévalences moyennes des hémoparasites et l'âge des oiseaux d'une part, et le sexe de ces derniers d'autre part.

Influence du poids et des saisons sur la distribution des hémoparasites

Les prévalences des infestations à Plasmodium/Haemoproteus, Leucocytozoon et
Trypanosoma en fonction du poids des oiseaux et des saisons sont représentées au Tableau 4.

Il se dégage de ce tableau que seuls les oiseaux de faibles poids (24,83 g en moyenne) ont présenté des infestations à Trypanosoma $s p$ (Tableau 4). Les oiseaux ayant un poids relativement plus élevé (40-49 g) ont été plus infestés par Plasmodium/Haemoproteus et Leucocytozoon. Quant aux saisons, les infestations par Plasmodium/Haemoproteus et Trypanosoma sont légèrement plus élevées en saison des pluies que pendant la saison sèche. Par contre, celles à leucocytozoon restent élevées en saison sèche (Tableau 4). Aucune différence significative n'a été observée entre les prévalences moyennes d'infestation et les différentes saisons.

Les infestations simples à Plasmodium/HaemoProteus ont été les plus fréquentes $(45,16 \%)$, suivit des infestations doubles à Plasmodium/HaemoProteus et Leucocytozoon (9,68\%) (Figure 3). Un seul sujet a présenté une infestation triple.

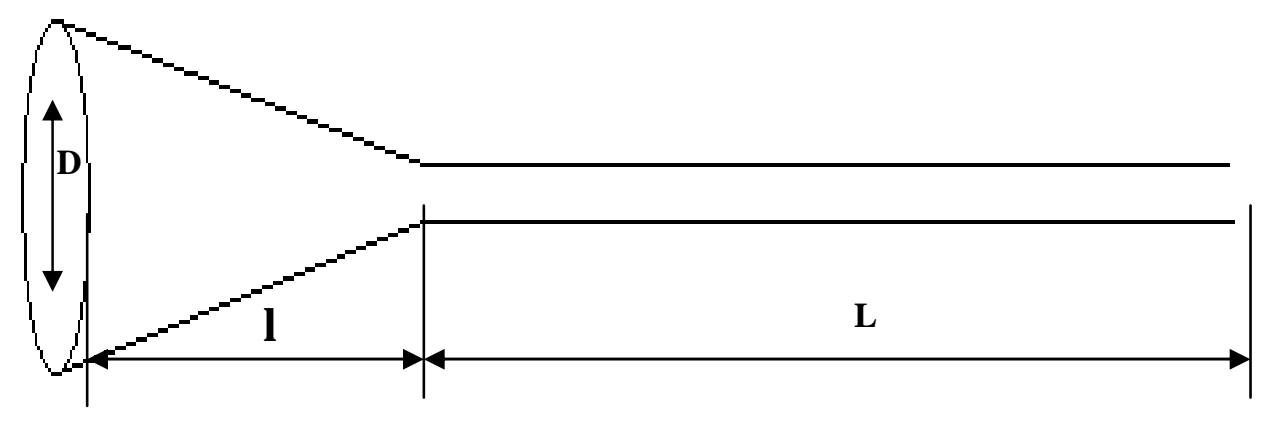

$D=$ Diamètre d'ouverture du nid=10 $\mathrm{cm} \quad l=35 \mathrm{~cm}$

$L=$ Longueur du bambou, variable en fonction de la hauteur des colonies

Figure 2: Piège de capture des oiseaux à l'extrémité d'un bambou. 


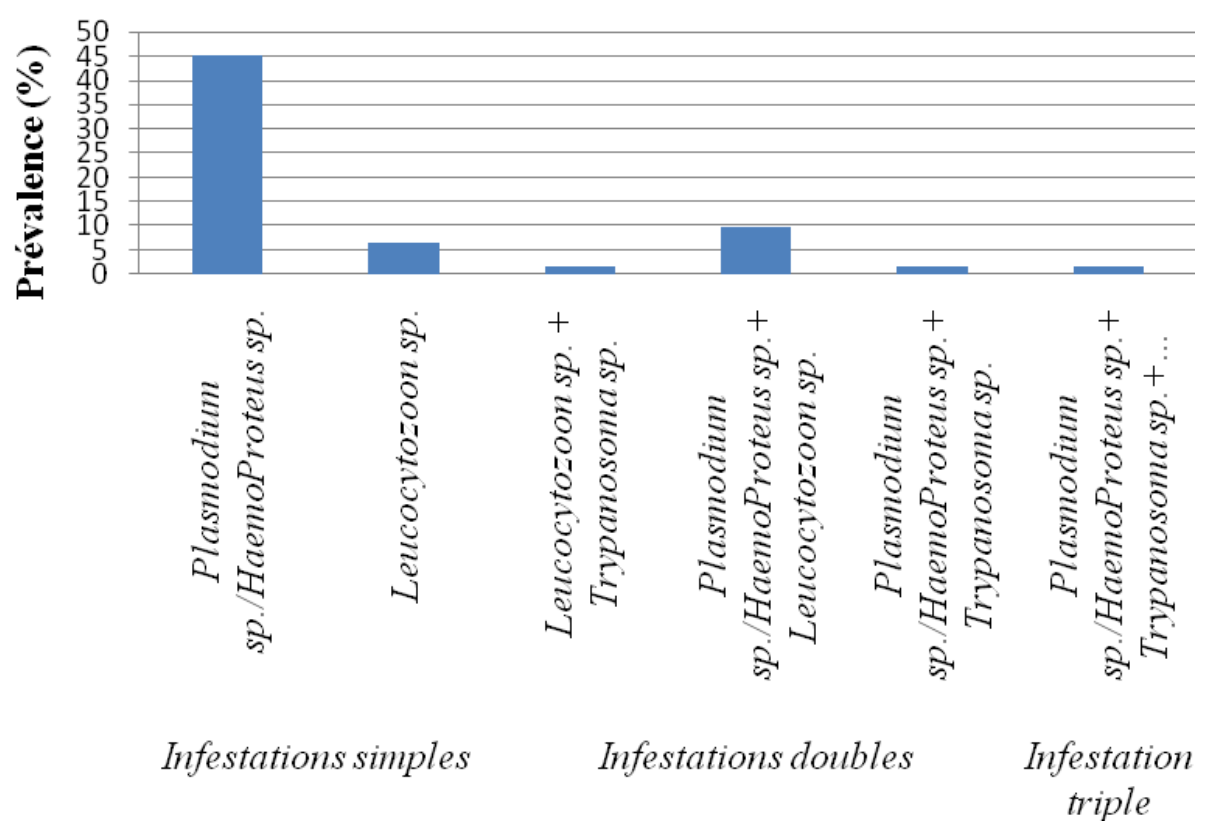

Figure 3 : Associations parasitaires pour les infestations à hémoparasites.

Tableau 2: Prévalences des hémoparasites selon les sites de capture.

\begin{tabular}{|c|c|c|c|c|c|c|c|c|}
\hline \multirow{2}{*}{$\begin{array}{l}\text { Sites de } \\
\text { capture }\end{array}$} & \multicolumn{2}{|c|}{$\begin{array}{c}\text { Plasmodium/ } \\
\text { Haemoproteus }\end{array}$} & \multicolumn{2}{|c|}{ Leucocytozoon } & \multicolumn{2}{|c|}{ Trypanosoma } & \multicolumn{2}{|c|}{$\begin{array}{c}\text { Infection totale à } \\
\text { hémoparasites }\end{array}$} \\
\hline & $\mathbf{I} / \mathbf{E}$ & Prév (\%) & $\mathbf{I} / \mathbf{E}$ & Prév (\%) & $\mathbf{I} / \mathbf{E}$ & Prév (\%) & $\mathbf{I} / \mathbf{E}$ & Prév (\%) \\
\hline Regie & $8 / 16$ & 50,00 & $5 / 16$ & 32,25 & $2 / 16$ & 12,50 & $10 / 16$ & 62,50 \\
\hline Tsinkop & $4 / 10$ & 40,00 & $3 / 10$ & 30,00 & $0 / 10$ & 0,00 & $6 / 10$ & 60,00 \\
\hline Foreké & $5 / 8$ & 62,50 & $1 / 8$ & 12,50 & $0 / 8$ & 0,00 & $5 / 8$ & 62,50 \\
\hline Campus A & $10 / 16$ & 62,50 & $1 / 16$ & 6,25 & $0 / 16$ & 0,00 & $11 / 16$ & 68,75 \\
\hline Mingouh & $6 / 7$ & 85,71 & $1 / 7$ & 14,29 & $1 / 7$ & 14,29 & $6 / 7$ & 85,71 \\
\hline Paidground & $3 / 5$ & 60,00 & $1 / 5$ & 20,00 & $0 / 5$ & 0,00 & $3 / 5$ & 60,00 \\
\hline Total & $36 / 62$ & 58,06 & $12 / 62$ & 19,35 & $3 / 62$ & 4,84 & $41 / 62$ & 66,13 \\
\hline
\end{tabular}


Tableau 3: Prévalences des hémoparasites par âge et par sexe d'oiseaux.

\begin{tabular}{|c|c|c|c|c|c|c|}
\hline \multirow[t]{2}{*}{ Age } & \multicolumn{2}{|c|}{$\begin{array}{c}\text { Plasmodium / Haemo- } \\
\text { Proteus }\end{array}$} & \multicolumn{2}{|c|}{ Leucocytozoon } & \multicolumn{2}{|c|}{ Trypanosoma } \\
\hline & $\mathrm{I} / \mathrm{E}$ & Prév (\%) & $\mathrm{I} / \mathrm{E}$ & Prév (\%) & $\mathrm{I} / \mathrm{E}$ & Prév (\%) \\
\hline Adulte & $27 / 45$ & 60,00 & $8 / 45$ & 17,78 & $1 / 45$ & 2,22 \\
\hline Jeune & $9 / 17$ & 52,94 & $4 / 17$ & 23,53 & $2 / 17$ & 11,76 \\
\hline Sexe & & & & & & \\
\hline Mâle & $17 / 26$ & 65,38 & $6 / 26$ & 23,08 & $0 / 26$ & 0,00 \\
\hline Femelle & $19 / 36$ & 52,78 & $6 / 36$ & 16,67 & $3 / 36$ & 8,33 \\
\hline
\end{tabular}

Tableau 4 : Prévalences de différents hémoparasites en fonction du poids et des saisons.

\begin{tabular}{lcccccc}
\hline & \multicolumn{2}{c}{$\begin{array}{c}\text { Plasmodium } \mathbf{s p} \text { / Haemo- } \\
\text { Proteus }\end{array}$} & \multicolumn{2}{c}{ Leucocytozoon } & \multicolumn{2}{c}{ Trypanosoma } \\
\cline { 2 - 6 } & I/E & Prév (\%) & I/E & Prév $(\%)$ & I/E & Prév (\%) \\
\hline Poids (g) & & & & & & \\
$20-29$ & $20 / 34$ & 58,82 & $5 / 34$ & 14,71 & $3 / 34$ & 8,82 \\
$30-39$ & $12 / 23$ & 52,17 & $5 / 23$ & 21,74 & $0 / 23$ & 0,00 \\
$40-49$ & $4 / 5$ & 80,00 & $2 / 5$ & 40,00 & $0 / 5$ & 0,00 \\
Saisons & & & & & & \\
Saison des pluies & $10 / 16$ & 62,50 & $2 / 16$ & 12,50 & $1 / 16$ & 6,25 \\
Saison sèche & $26 / 46$ & 56,52 & $10 / 46$ & 21,74 & $2 / 46$ & 4,35 \\
\hline I= Nombre d'individus infectés & E = Nombre d'individus examinés & Prév = Prévalence &
\end{tabular}

\section{DISCUSSION}

La prévalence totale de $66,13 \%$ des infestations à hémoparasites rencontrées chez le tisserin villageois dans la ville de Dschang et ses environs est élevée comparée aux résultats obtenus chez d'autres familles d'oiseaux y compris la famille des Ploceidae à laquelle appartient $P$. cucullatus. Elle était de $35,9 \%$ pour les oiseaux sauvages de Madagascar (Rahimanga et al., 2002) et $28,6 \%$ pour les oiseaux de la région équatoriale de l'Afrique de l'Ouest où seuls 3 pays (Cameroun, Guinée Equatoriale et Côte
d'Ivoire) ont fait l'objet des prélèvements (Sehgal et al., 2005).

Les informations sur les hémoparasites des tisserins villageois sont rares. La prévalence totale d'hémoparasites observée chez cette espèce se rapproche des résultats des travaux menés au Cameroun par Sehgal et al. (2005), où la prévalence des Ploceidae nidifiant en colonie (Ploceus nigerrimus et Quelea erythrops) était de 62,5\%. Cette prévalence élevée d'hémoparasites chez $P$. cucullatus serait dû au comportement de l'oiseau qui mène une vie coloniale, de 
préférence à proximité des points d'eau où il y a abondance des vecteurs.

La prévalence de Leucocytozoon $s p$. obtenue chez $P$. cucullatus $(19,35 \%)$ est faible par rapport à celle obtenue chez $P$. nigerrimus (38\%) à Sangmbemgue au Sud Cameroun pendant la saison des pluies (Sehgal et al., 2005). Cette différence serait liée à la saison ou à la spécificité de l'hôte. La grande partie de notre travail a été effectuée en saison sèche. L'influence des saisons a déjà été observée dans la transmission des hémoparasites avec des prévalences élevées en saison des pluies (Atkinson et al., 1993). La capacité vectrice des vecteurs est maximale en saison des pluies (Rahimanga et al., 2002).

Aucune microfilaire n'a été détectée, cette absence serait due à la période de prélèvement, la moindre susceptibilité de l'espèce à ce parasite, la rareté des insectes vecteurs, ou la faible prévalence de ce nématode chez le tisserin villageois à Dschang. Chez les oiseaux, le cycle biologique des microfilaires est inconnu (Rahimanga et al., 2002).

Les vecteurs impliqués dans la transmission d'hémoparasites d'oiseaux sont presque inconnus. Plusieurs types de vecteurs sont supposés contribuer à la transmission (Rahimanga et al., 2002). Mais il n'est pas du tout évident que les vecteurs avérés dans le reste du monde soient les même au Cameroun. Les infections à hémoparasites chez $P$. cucullatus semblent bénignes. Certaines souches de Leucocytozoon peuvent être mortelles (Rahimanga et al., 2002), certains Plasmodium ont entraîné une forte mortalité chez les pingouins (Garnham, 1966) cité par Rahimanga et al. (2002) et chez les oiseaux d'espèce forestière à Hawaï (Atkinson et al., 1993). Peu de données sont disponibles concernant les infestations à trypanosomes et à microfilaires mais ces parasites ne semblent pas être responsables d'importants effets pathogènes.

La prévalence élevée d'hémoparasites à Mingouh est liée à la saison (saison des pluies). Les variations de la prévalence observées en fonction des sites de capture sont liées à la saison pour les Plasmodium sp/Haemoproteus sp et les Trypanosoma. Les captures étaient effectuées dans chaque site à des périodes différentes puisque les six sites de nidification des tisserins villageois présentaient les mêmes caractéristiques. Dans certains sites, les oiseaux ont été capturés à la fois en saison sèche et en saison des pluies (Régie, Tsinkop, Campus A) tandis que dans les autres sites, les captures étaient effectuées seulement en saison des pluies (Paidground et Mingouh) parce que les oiseaux y étaient présents seulement pendant cette période.

Ces prévalences sont légèrement élevées en début de saison de pluies (mi-mars à avril) par rapport à la saison sèche (minovembre à mi-mars). Ceci semble logique par rapport aux vecteurs et aux importantes variations de leurs capacités vectrices, maximales en saison des pluies (Rahimanga et al., 2002). Cette influence des saisons a déjà été observées précédemment (Atkinson et al., 1993). Contrairement aux Plasmodium sp/Haemoproteus sp et les Trypanosoma, la prévalence des Leucocytozoon est légèrement élevée en saison sèche qu'en saison des pluies. Cette observation est inattendue, certains facteurs autre que la saison influencerait la transmission de ce type de parasites chez $P$. cucullatus (altitude, saison sèche pas très rude, présence constante d'eau).

Les oiseaux de sexe mâle ont tendance à être plus parasités par Plasmodium sp/Haemoproteus sp, Leucocytozoon sp. Ceci pourrait être dû à une surexposition à la transmission à cause des déplacements accrus pour les mâles favorisant les contacts avec les vecteurs.

\section{Conclusion}

Le tisserin gendarme, oiseau le plus commun et le plus abondant de la famille des Ploceidae hébergent bon nombre d'hémoparasites. A cet effet, leurs multiples déplacements autour des villes et villages favoriseraient la distribution des parasites qu'ils portent dans la nature par l'intermédiaire des arthropodes, agents 
vecteurs des maladies. Les oiseaux sont également des réservoirs de nombreuses zoonoses parasitaires et microbiennes. En effet, leur proximité (tisserin) avec les humains et autres animaux pourrait entraîner un échange d'hémoparasites par la piqûre des moustiques. Il est connu que certains moustiques de la familles des Culicidae (Aedes), responsable de la transmission de Plasmodium dans la présente étude, sont aussi des vecteurs pour de nombreuses maladies virales telles que : la dengue, la fièvre jaune, etc. Les moustiques assurent aussi la transmission de nombreux autres virus à l'origine des maladies telle que: la fièvre du Nil occidental, la fièvre de Mayaro, l'encéphalite équine est-américaine, etc. Les tisserins constituent de ce fait un risque significatif pour la santé humaine et animale. Cette étude s'insère aussi dans un programme de surveillance écologique, microbiologique dont le but est de comprendre les mécanismes qui conditionnent l'apparition d'une situation épidémiologique liée à l'agent pathogène luimême, son cycle naturel, l'hôte ou l'environnement.

\section{REMERCIEMENTS}

Le fond de carte présent dans ce document nous a été aimablement confectionné par le CEREHT (Centre de Recherche sur les Hautes Terres) de la Faculté des Lettres et des Sciences Humaines (FLSH) de l'Université de Dschang. Ce travail a été réalisé au Laboratoire de Biologie et d'Ecologie Appliquée (LABEA) de la Faculté des Sciences de l'Université de Dschang (Cameroun). Nous remercions tous les enseignants du Département de Biologie Animale et ceux de Physiologie Animale de la FASA (Faculté d'Agronomie et des Sciences Agricoles) de l'Université de Dschang pour leur contribution à l'accomplissement de ce travail.

\section{REFERENCES}

Atkinson CT, Dusek RJ, Iko WM. 1993. Epidemic pox and malaria in native forest birds. Hawaï's Forest and Wildlife, 8: 10.

Barroca M. 2005. Hétérogénéité des relations parasites-oiseaux : importance écologique et rôle évolutif. Thèse de Doctorat, Université de Bourgogne, 21000 Dijon. $185 \mathrm{p}$.

Clayton DH, Moore J. 1997. Host-Parasite Evolution. General Principles and Avian Models. Oxford University Press: New York.

Daszak P, Cunningham AA, Hyatt AD. 2000. Emerging infectious diseases of wildlife threats to biodiversity and human health. Science, 287: 443-449.

Fry CH, Keith S. 2004. The Birds of Africa (Vol 7). Christopher Helm: London.

Kirkpatrick CE, Smith TB. 1988. Blood parasites of birds in Cameroun. $J$. Parasitol., 74: 1009-1013.

Murphy FA. 1998. Emerging zoonoses. Emerg Infec Dis., 4: 429-435.

OMS (Organisation Mondial de la Santé). 1982. Technique de Base pour le Laboratoire Médical, par Etienne Lévy Lambert. OMS.

Pennycott TW, Ross HM, Pennycott TW, Hopkins GM, McLaren IM. 1998. Causes of death of wild birds of the family Fringillidae in Britain. Veterinary Record, 143: $155-158$.

Rahimanga V, Soula F, Raherilalao MJ, Goodman SM, Sadones H, Tall A, Randrianarivelojosia M, Raharimalala L, Duchemin JB, Ariey F, Robert V. 2002. Hémoparasites des oiseaux sauvages à Madagascar. Arch Inst Pasteur de Madagascar, 68(1-2) : 90-99.

Sehgal RNM, Jones HI, Smith TB. 2005. Blood parasites of some West African rainforest birds. J. Vet. Med. Sci., 67(3): 295-301.

Valkiūnas G. 2005. Avian malaria parasites and other Haemasporidae. CRC Press: New York. 